adult population (aged 55-75 years) studied. We have prospectively studied 45 stroke patients (aged 26 to 65 years) for psychiatric morbidity. The most common disorder was depression (in $78 \%$ of the patients), followed by generalised disorder (in $17 \%$ ). Younger age, physical disability (resulting in occupational and social dysfunction) and past history of stroke were strongly correlated with depression. Vascular disease has been found to be associated with a more prolonged duration of depression (Hickie \& Scott, 1998), but in our sample $52 \%$ of the patients with depression recovered within 3-6 months of treatment. However, two patients who were unemployed when they were disabled by stroke did have depression of prolonged duration. Uncontrolled hypertension (moderate to severe) was associated with the presence of generalised anxiety disorder. The role of medication (especially beta-blockers, calcium channel blockers and sedatives) in producing depression is an important variable and could not be ruled out in six patients. Although laterality of brain lesion (i.e. left hemispheric lesion) and risk of depression have been reported (Robinson \& Price, 1982), the subject remains controversial and we did not find any such association. A detailed prospective study on a larger sample of patients from all age groups and different socio-demographic backgrounds is needed to establish the association of depression with various demographic and vascular risk factors for stroke.

Hickie, I. \& Scott, E. (1998) Late-onset depressive disorders: a preventable variant of cerebrovascular disease? Psychological Medicine, 28, 1007-1013.

Robinson, R. C. \& Price, T. R. (1982) Post-stroke depressive disorders: a follow-up study of 103 patients. Stroke, 13, 635-64l.

Stewart, R., Prince, M., Richards, M., et al (200I) Stroke, vascular risk factors and depression. Crosssectional study in a UK Caribbean-born population. British Journal of Psychiatry, 178, 23-28.

M. S. Bhatia, R. Chandra Department of Psychiatry, University College of Medical Sciences, Dilshad Garden, Delhi II0095, India

\section{Somatoform disorders: a topic for education}

Bass et al (2001) believe that somatoform disorders are ignored by psychiatrists and health service planners because of the nature of diagnostic practice, a current preoccupation with only "serious mental illness", limited experience of patients with medically unexplained symptoms in general hospital settings, and stigma. They do not mention whether they have found an increasing fear of litigation to be another contributing factor. Currently, it appears to play a part in delaying referral to psychological services while the patient is exhaustively investigated for any physical pathology. Any comment they might make regarding this practice would be of interest.

Certainly, as they mention, a lack of training of non-psychiatric practitioners in this area contributes greatly to non-referral within the general hospital setting. We would, however, dispute their comment that psychiatrists working in this area find that patients with somatoform disorders "comprise between one-third and one-half of all referrals to the liaison psychiatry service". A review carried out several years ago of the nature of referrals to the consultation-liaison services of two general hospitals in Dublin City (Cullivan et al, 1997) suggests a much smaller number of such referrals. Over a 6-month period 491 patients were referred and patients with diagnoses falling into categories F40-F48 of ICD-10 (neurotic, stress-related and somatoform disorders) accounted for only $12 \%$ of referrals in one hospital and $15 \%$ in the other. As a significant number of the patients in these categories were suffering from adjustment disorders, the numbers diagnosed with somatoform disorders, formed an even smaller percentage of all referrals.

It is worth noting that these were the diagnostic categories provided by the psychiatrists who assessed these patients. The reason for the referrals given by the medi$\mathrm{cal} /$ surgical teams was "no organic cause for symptoms found" in just $1.7 \%$ of cases in one hospital and $10.2 \%$ in the other. Perhaps somatoform disorders are even more neglected than previously thought? Education of both psychiatric and nonpsychiatric personnel regarding these disorders would appear to be in need of urgent review.

Bass, C., Peveler, R. \& House, A. (200I) Somatoform disorders: severe psychiatric illnesses neglected by psychiatrists. British Journal of Psychiatry, 179, II-14.

Cullivan, R., Durkin, I. \& Kelly, G. (1997)

Consultation-liaison psychiatry - a comparison of two services. Irish Journal of Medical Science, 166, 23-24.

R. Cullivan Department of Psychiatry, St Camillus Unit, St Vincent's Hospital, Donnybrook, Dublin 4, Ireland
We read with interest Bass et al's (2001) review on somatoform disorders. Although the authors usefully pointed out that these disorders are common and cause severe disability, we were dismayed to find that, ironically, they neglected to mention one of the more common somatoform disorders: body dysmorphic disorder (BDD; also known as dysmorphophobia). A distressing or impairing preoccupation with an imagined or slight defect in appearance, BDD has reported rates in the community of $0.7-2.3 \%$ (Phillips, 2001). People with this disorder commonly present to psychiatrists, dermatologists, cosmetic surgeons and other physicians (Phillips \& Castle, 2001).

Body dysmorphic disorder causes severe distress and marked impairment in functioning (Veale et al, 1996; Phillips, 2001). A high proportion of patients require hospitalisation, become housebound and/ or attempt suicide. Completed suicide has been reported in both psychiatric and dermatology settings. Mental-health-related quality of life is poorer than that reported for patients with depression, obsessivecompulsive disorder and a variety of physical illnesses, including recent myocardial infarction and type II diabetes.

Like the other somatoform disorders, BDD is often neglected by psychiatrists. The diagnosis is usually missed in mental health settings (Phillips \& Castle, 2001). This is unfortunate, because a majority of these patients request and receive nonpsychiatric treatments, such as dermatological treatment and surgery, which are usually ineffective. Many patients consult numerous physicians, request extensive work-ups, and pressure dermatologists and surgeons to provide unsuitable and ineffective remedies. Some patients, in desperation, even perform their own surgery. As one dermatologist stated, "The author knows of no more difficult patients to treat than those with body dysmorphic disorder" (Cotterill, 1996).

The good news is that emerging data indicate that a majority of these patients can be successfully treated with selective serotonin reuptake inhibitors or cognitivebehavioural therapy (Phillips, 2001). It is important that psychiatrists and other physicians screen patients for this disorder so that effective treatment can be provided. Body dysmorphic disorder is a severe psychiatric illness that we cannot afford to neglect.

Bass, C., Peveler, R. \& House, A. (200I) Somatoform disorders: severe psychiatric illnesses neglected by psychiatrists. British Journal of Psychiatry, 179, 11-14. 
Cotterill, J. A. (1996) Body dysmorphic disorder. Dermatology Clinics, 14, 457-463.

Phillips, K. A. (200I) Body dysmorphic disorder. In Somatoform and Factitious Disorders (ed. K. A. Phillips), pp. 67-94. Washington, DC: American Psychiatric Press.

\& Castle, D. J. (200I) Body dysmorphic disorder. Disorders of Body Image (eds D. J. Castle \& K. A. Phillips). Petersfield: Wrightson Biomedical, in press.

Veale, D., Boocock, A., Gournay, K., et al (1996) Body dysmorphic disorder. A survey of fifty cases. British Journal of Psychiatry, 169, 196-201.

K. A. Phillips Butler Hospital, Brown University School of Medicine, Providence, Rhode Island, USA

D. J. Castle Fremantle Hospital, University of Western Australia, PO Box 480, Fremantle, WA 6959, Australia

\section{Advice for authors is premature}

In their recent article Patel \& Sumathipala (2001) lament the low level of international representation in high-impact psychiatry journals and argue that such a phenomenon is curtailing the development of the psychiatric discipline in both developed and developing countries. Although I agree with the basic argument put forward, some of the advice given to prospective authors is, at best, premature. To be more specific, they explicitly advise authors from countries outside the 'Euro-American' group (Western Europe, North America and Australia/New Zealand) to submit their manuscripts to the three high-impact European psychiatric journals (British Journal of Psychiatry, Acta Psychiatrica Scandinavica and Psychological Medicine), rather than to the three high-impact American psychiatric journals (American Journal of Psychiatry, Archives of General Psychiatry and Schizophrenia Bulletin), because the former publish a higher proportion of articles from 'rest-of-the-world' (RoW) authors. While this may be so, and indeed their data suggest that it is, it does not necessarily follow that such authors will improve their chances of publication by submitting to the three European journals in preference to the three American ones. Such authors should be concerned with differential acceptance rates rather than with the proportion of published papers by RoW authors. Although no acceptance rate data were provided by the three American journals, data on the three European journals indicated a much lower acceptance rate for RoW authors than for Euro-American ones (the fact that the three American journals refused to provide acceptance rate data should not be assumed to indicate that they show an even greater bias). Given these data, it would seem wrong to suggest that RoW authors should favour the three European journals when submitting manuscripts for publication. Such advice should perhaps be reserved until the data are more conclusive.

Patel, V. \& Sumathipala, A. (200I) International representation in psychiatric literature. Survey of six leading journals. British Journal of Psychiatry, 178 406-409.

G. J. Faunce Department of Psychiatry, University of Sydney, NSW 2006, Australia

\section{Chromosome 22qll deletions and severe learning disability}

In a previous study (Murphy et al, 1998) we identified 74 patients thought to be at risk of velo-cardio-facial syndrome (VCFS) from 265 hospitalised individuals with learning disability. We screened these 74 people and found two cases of VCFS, giving a minimum prevalence of $0.7 \%$ in the entire sample. Recently, we found a further individual with VCFS among the 191 individuals who were not selected for screening. This gives a revised minimum prevalence estimate of $1.1 \%$.

The patient, a 50-year-old Caucasian female with severe learning disabilities, had no reported complications arising during gestation, delivery or early childhood. Developmental milestones were globally delayed. She presented to the psychiatric services as a teenager with a 12-month history of becoming withdrawn and subdued, having lost some previously learned skills and displaying outbursts of bizarre behaviour and aggression. She was subsequently admitted on several occasions and was definitively admitted as a longterm patient within 2 years of initial presentation, owing to her enduring challenging behaviour. At this time, her mental age was assessed to be between 2 and 4 years and she had not developed any effective speech or signing. She remained in hospital for over 30 years, then was discharged to a staffed house in the community. While in hospital she remained largely quiet, timid and withdrawn. However, there were episodes of prolonged challenging behaviour, during which time she became suspicious, would scream, cry and make unintelligible noises, often accompanied by physical aggression. These episodes were treated with a variety of behavioural modification strategies, augmented with various antipsychotic preparations with partial success. No formal diagnosis of psychotic illness was made and she was therefore not selected for chromosome 22q11 deletion studies as she did not meet screening criteria (Murphy et al, 1998).

In 1999 she was readmitted to the local assessment and treatment unit because of aggression, agitation, deterioration in her skills and bizarre behaviour including ambitendence, vocalising and gesturing as if responding to abnormal perceptions. It was felt that these symptoms were suggestive of a psychotic disorder and she was successfully treated with an atypical antipsychotic. A provisional diagnosis of schizophrenia was made, although one cannot be categorical in individuals lacking effective communication skills (Royal College of Psychiatrists, 2001). Fluorescence in situ hybridisation (FISH) analysis confirmed a deletion or chromosome 22q11.

In our 1998 study, we concluded that chromosome 22q11 deletions appear to be aetiologically significant in a proportion of individuals with idiopathic learning disability, especially in those with mild learning disability and psychosis. The importance of the patient described above is that she has severe rather than mild learning disability and that, despite presenting with seriously challenging behaviour at the time of the study, she was not diagnosed as having a formal psychotic illness.

Although individuals with VCFS are characterised predominantly by borderline or mild learning disability, it is important for clinicians to be aware that occasionally such individuals may present with more severe learning disabilities, as in this case. We suggest that any individual with moderate or severe learning disabilities with a history of bizarre or uncharacteristic challenging behaviour, even in the absence of a formal diagnosis of psychosis, should be referred for chromosome 22q11 deletion studies.

Murphy, K. C., Jones, R. G., Griffiths, E., et al (1998) Chromosome 22qll deletions. An under-recognised cause of idiopathic learning disability. British Journal of Psychiatry, 172, 180-183.

Royal College of Psychiatrists (200I) DC-LD Diagnostic Criteria for Psychiatric Disorders for use with Adults with Learning Disabilities/Mental Retardation (Occasional Paper OP48). London: Gaskell.

R. G. Jones, J. Morley-Canellas Learning Disability Directorate, Bro Morgannwg NHS Trust, Treseder Way, Caerau, Cardiff CF5 5WF, UK 